\title{
On the derivation of explicit two-step peer methods ${ }^{\text {th }}$
}

\author{
M. Calvo ${ }^{\mathrm{a}}$, J.I. Montijano ${ }^{\mathrm{a}, *}$, L. Rández ${ }^{\mathrm{a}}$, M. Van Daele ${ }^{\mathrm{b}}$ \\ ${ }^{a}$ Departamento Matemática Aplicada, Universidad de Zaragoza, 50009-Zaragoza, Spain. \\ ${ }^{b}$ Vakgroep Toegepaste Wiskunde en Informatica, Universiteit Gent, Krijgslaan 281-S9, \\ B9000 Gent, Belgium.
}

\begin{abstract}
The so called peer methods for the numerical solution of Initial Value Problems (IVP) in ordinary differential systems were introduced and later applied to different types of problems by R. Weiner, B.A. Schmitt and coworkers in a series of papers [10], [11], [12], [13], [16], [17], [18] as an alternative to classical Runge-Kutta (RK) and multistep methods attempting to combine the advantages of these two classes of methods. In particular, peer type methods have been proposed in [11], [12], [13] for the numerical solution of IVPs in parallel computers. More recently, several explicit two step peer methods for non stiff systems proposed in [17], [18] have been proved to be competitive with standard RK methods in a wide selection of test problems.

The aim of this paper is to propose an alternative procedure to construct families of explicit two step peer methods in which the available parameters appear in a transparent way. This allows us to obtain families of fixed stepsize $s$ stage methods with stage order $2 s-1$, which provide dense output without extra cost, depending on some free parameters that can be selected taking into account the stability properties and leading error terms. A study of the extension of these methods to variable stepsize is also carried out. Optimal $s$ stage methods with $s=2,3$ are derived.
\end{abstract}

Keywords: Peer methods, two-step methods, IVP, stability 2000 MSC: 65L05, 65L07

\section{Introduction}

We consider the numerical solution of (non stiff) IVPs for a differential system

$$
\frac{\mathrm{d}}{\mathrm{d} t} y(t)=f(t, y(t)), \quad y\left(t_{0}\right)=y_{0} \in \mathbb{R}^{m},
$$

\footnotetext{
This work was supported by project DGI-2007-MTM2007-67530-C02-01

* Corresponding author

Email addresses: calvo@unizar.es (M. Calvo), monti@unizar.es (J.I. Montijano), randez@unizar.es (L. Rández), Marnix.VanDaele@ugent.be (M. Van Daele)
} 
where $f: \mathbb{R} \times \mathbb{R}^{m} \rightarrow \mathbb{R}^{m}$ is assumed to be sufficiently smooth so that for all $y_{0}$ there is a unique smooth solution to the right of $t_{0}$.

To introduce the $s$-stage two-step peer methods, let $c_{j}, j=1, \ldots, s$ be a given admissible set of nodes, i.e.

$$
\left|c_{i}-c_{j}\right| \notin\{0,1\} \text { for all } i \neq j \text {. }
$$

We assume that for any given step size $h>0$ there is a starting procedure that allows us to obtain (sufficiently accurate) approximations

$$
Y_{0, j} \simeq y\left(t_{0, j}\right), \quad f_{0, j}=f\left(t_{0, j}, Y_{0, j}\right), \quad j=1, \ldots, s
$$

to the solution of (1) and to the vector field at the internal grid points $t_{0, j}=t_{0}+$ $c_{j} h, j=1, \ldots s$ in the first step interval $\left[t_{0}, t_{1}=t_{0}+h\right]$. Then an $s$-stage explicit two-step peer method is an algorithm that computes new approximations

$$
Y_{1, j} \simeq y\left(t_{1, j}\right), \quad f_{1, j}=f\left(t_{1, j}, Y_{1, j}\right), \quad j=1, \ldots, s
$$

to the solution of (1) and the vector field at the internal grid points $t_{1, j}=$ $t_{1}+c_{j} h, j=1, \ldots s$ of the next step $\left[t_{1}, t_{2}=t_{1}+h\right]$ by means of the equations

$$
Y_{1, j}=\sum_{k=1}^{s} a_{j k} Y_{0, k}+h \sum_{k=1}^{s} b_{j k} f_{0, k}+h \sum_{k=1}^{j-1} r_{j k} f_{1, k}, \quad j=1, \ldots, s
$$

where $A=\left(a_{j k}\right), B=\left(b_{j k}\right), R=\left(r_{j k}\right) \in \mathbb{R}^{s \times s}$ are given matrices that define the method. $A$ and $B$ are full matrices and $R$ is strictly lower triangular.

It must be noticed that integration methods that combine information about the solution in two consecutive steps have been considered for a long time as can be seen in the earlier publications [2], [7], [8] and [16]. In particular, the General Linear Methods introduced by J.C. Butcher in [2] as a generalization of linear multistep (multivalue) methods and Runge-Kutta (multistage) methods also contain the explicit peer two-step methods.

Weiner and Schmitt and co-workers have provided for some special $A$-matrix methods of type (3) of different orders [10], [17], [18] that are competitive with the standard integrators in use.

Putting $\mathbf{e}=(1, \ldots, 1)^{T} \in \mathbb{R}^{s}, \mathbf{c}=\left(c_{1}, \ldots, c_{s}\right)^{T}$ and

$$
\mathbf{Y}_{k}=\left(\begin{array}{c}
Y_{k, 1} \\
Y_{k, 2} \\
\vdots \\
Y_{k, s}
\end{array}\right), \quad f\left(t_{k} \mathbf{e}+h \mathbf{c}, \mathbf{Y}_{k}\right)=\left(\begin{array}{c}
f\left(t_{k, 1}, Y_{k, 1}\right) \\
f\left(t_{k, 2}, Y_{k, 2}\right) \\
\vdots \\
f\left(t_{k, s}, Y_{k, s}\right)
\end{array}\right) \in\left(\mathbb{R}^{m}\right)^{s}
$$

equations (3) can be written in the matrix form

$$
\begin{aligned}
\mathbf{Y}_{1}= & \left(A \otimes I_{m}\right) \mathbf{Y}_{0}+h\left(B \otimes I_{m}\right) f\left(t_{0} \mathbf{e}+h \mathbf{c}, \mathbf{Y}_{0}\right) \\
& +h\left(R \otimes I_{m}\right) f\left(t_{1} \mathbf{e}+h \mathbf{c}, \mathbf{Y}_{1}\right)
\end{aligned}
$$


where $\otimes$ denotes the standard Kronecker product and $I_{m}$ is the unit matrix of order $m$. For our studies of order and stability it will be sufficient to consider the scalar case $(m=1)$ in which (5) becomes

$$
\mathbf{Y}_{1}=A \mathbf{Y}_{0}+h B f\left(t_{0} \mathbf{e}+h \mathbf{c}, \mathbf{Y}_{0}\right)+h R f\left(t_{1} \mathbf{e}+h \mathbf{c}, \mathbf{Y}_{1}\right) .
$$

As it has been pointed out in [11], [18] the method (5) is zero-stable if and only if the matrix $A$ has an eigenvalue $\lambda_{1}(A)=1$ and the remaining eigenvalues $\lambda_{j}(A), j=2, \ldots, s$ have modulus $\leq 1$ and those of modulus one correspond to simple elementary divisors. Hence, a safe stability requirement is to choose $A$ so that

$$
\lambda_{1}(A)=1, \quad \lambda_{j}(A)=0, \quad j=2, \ldots, s,
$$

because these conditions ensure the zero stability ([6], pp. 293). Remark that $\lambda_{1}(A)=1$ is a consequence of the preconsistency condition $A \mathbf{e}=\mathbf{e}$. Thus, Weiner and coworkers take in [18] a constant matrix $A$ of the form $A=\mathbf{e}_{s}^{T}+$ $Q W Q^{-1}$ where $W, Q$ are some special matrices such that $A \mathbf{e}=\mathbf{e}$ and $A$ has eigenvalues 1 (simple) and 0 and $\mathbf{e}_{i}=(0, \ldots, 1, \ldots, 0)^{T}$ is the $i$-th unit vector of the canonical basis of $\mathbb{R}^{s}$.

Here we will choose an alternative family of matrices $A$ of the form

$$
A=P^{-1} \widehat{A} P,
$$

with lower triangular constant $P$ and upper triangular constant $\widehat{A}$ given by

$P=\left(\begin{array}{ccccc}1 & 0 & \ldots & 0 & 0 \\ p_{21} & 1 & \ldots & 0 & 0 \\ p_{31} & p_{32} & \ldots & 0 & 0 \\ \vdots & \vdots & & \vdots & \vdots \\ p_{s-1,1} & p_{s-1,2} & \ldots & 1 & 0 \\ p_{s 1} & p_{s 2} & \ldots & p_{s, s-1} & 1\end{array}\right), \widehat{A}=\left(\begin{array}{ccccc}1 & \widehat{a}_{12} & \ldots & \ldots & \widehat{a}_{1 s} \\ 0 & 0 & \widehat{a}_{23} & \ldots & \widehat{a}_{2 s} \\ 0 & 0 & 0 & \widehat{a}_{34} & \ldots \\ \vdots & & & \vdots & \widehat{a}_{s-1, s} \\ 0 & \ldots & & 0 & 0\end{array}\right)$

that clearly satisfy (7). Note that the preconsistency condition $A \mathbf{e}=\mathbf{e}$ implies that $P \mathbf{e}=\mathbf{e}_{1}$.

The main reason to take $A$ with the form (8), (9) is that equations (6) can be written as

$$
P \mathbf{Y}_{1}=\widehat{A} P \mathbf{Y}_{0}+h \widehat{B} \operatorname{Pf}\left(t_{0} \mathbf{e}+h \mathbf{c}, \mathbf{Y}_{0}\right)+h \widehat{R} \operatorname{Pf}\left(t_{1} \mathbf{e}+h \mathbf{c}, \mathbf{Y}_{1}\right),
$$

with $\widehat{B}=P B P^{-1}$ and $\widehat{R}=P R P^{-1}$ where $\widehat{R}$ is lower triangular. Now for a given admissible set of nodes and a constant matrix $P$ with $P \mathbf{e}=\mathbf{e}_{1}$ the number of available parameters in the vector equation (10) is

$$
\frac{s(s-1)}{2} \text { in } \widehat{A}+s^{2} \text { in } \widehat{B}+\frac{s(s-1)}{2} \text { in } \widehat{R}=s(2 s-1),
$$


that are separated (linearly) into $s$ sets of $2 s-1$ at each stage. In fact, for the first stage the free parameters are those of the first row of the matrix

$$
(\widehat{A}|\widehat{B}| \widehat{R})=\left(\begin{array}{ccccc|ccc|cccc}
1 & \widehat{a}_{12} & \widehat{a}_{13} & \ldots & \widehat{a}_{1 s} & \widehat{b}_{11} & \ldots & \widehat{b}_{1 s} & 0 & & & \\
& 0 & \widehat{a}_{23} & \ldots & \widehat{a}_{2 s} & \widehat{b}_{21} & \ldots & \widehat{b}_{2 s} & \widehat{r}_{21} & 0 & & \\
& \vdots & \vdots & \vdots & \vdots & \vdots & \vdots & \vdots & \vdots & \vdots & \vdots & \\
& & & & 0 & \widehat{b}_{s 1} & \ldots & \widehat{b}_{s s} & \widehat{r}_{s 1} & \ldots & \widehat{r}_{s, s-1} & 0
\end{array}\right),
$$

for the second stage those of the second row and the same for the remaining stages. Because of this, in our order studies we may consider independently the order conditions of each stage of the transformed method (10). This simplifies considerably the derivation of particular explicit methods.

Note that if $A$ has the above spectrum (7) there exists a non singular real matrix $S$ that transforms $A$ to the Jordan canonical form i.e.

$$
A=S J S^{-1} \quad \text { with } J=\left(\begin{array}{c|cccc}
1 & 0 & 0 & \ldots & 0 \\
\hline 0 & 0 & \alpha_{2} & \ldots & 0 \\
0 & & & \ddots & \vdots \\
\vdots & & & & \alpha_{s-1} \\
0 & & & & 0
\end{array}\right),
$$

with $\alpha_{j}$ either 0 or else 1 . Now if $S$ possesses a LU decomposition without pivoting, $S=L U$, with unit diagonal elements in $L$, then $A$ can be written in the form

$$
A=L\left(U J U^{-1}\right) L^{-1},
$$

and it can be checked that the matrix $U J U^{-1}$ has the same zeroes as $\widehat{A}$ of $(9)$. Hence, even though not all matrices $A$ satisfying the spectral assumption (7) can be written in the form (8), (9), those for which the corresponding $S$ admits a LU decomposition without pivoting can.

For our (absolute) stability studies of the methods (6) we apply them to the scalar test equation $y^{\prime}=\lambda y$, where $\lambda$ is a complex constant. Putting $z=\lambda h$, (6) becomes

$$
\mathbf{Y}_{1}=(I-z R)^{-1}(A+z B) \mathbf{Y}_{0}
$$

or equivalently

$$
\mathbf{Y}_{1}=P^{-1} M(z) P \mathbf{Y}_{0}
$$

with $M(z)=(I-z \widehat{R})^{-1}(\widehat{A}+z \widehat{B}) \in \mathbb{C}^{s \times s}$. Then the stability region $S$ is the set of all $z \in \mathbb{C}$ such that all eigenvalues of $M(z)$ satisfy $\left|\lambda_{j}(M(z))\right| \leq 1$ and those with $\left|\lambda_{j}(M(z))\right|=1$ correspond to simple divisors in Jordan's canonical form.

\section{Order conditions}

In standard explicit RK methods the difference $y\left(t_{n}+h\right)-y_{n+1}$ defines the order of accuracy. Here, none of the $c_{i}$ needs to be 1 , and moreover we must 
take into account the order of accuracy of all stages $y\left(t_{1} \mathbf{e}+h \mathbf{c}\right)-\mathbf{Y}_{1}$, i.e. the so called stage order of the method. This is the motivation of the following definition of stage order:

For the method (6) with the matrix $A$ given by (8), (9) we introduce the linear $s$-dim valued vector operator $\mathcal{L}[y(t) ; h]$ defined by

$$
\mathcal{L}[y(t) ; h] \equiv \mathbf{Y}(t+h)-A \mathbf{Y}(t)-h B \mathbf{Y}^{\prime}(t)-h R \mathbf{Y}^{\prime}(t+h),
$$

with $\mathbf{Y}(t)=y(t \mathbf{e}+h \mathbf{c}) \equiv\left(y\left(t+c_{1} h\right), \ldots, y\left(t+c_{s} h\right)\right)^{T}$.

Definition 1 (Stage Order). For a given admissible set of nodes the method (6),(8),(9) has stage order $p$ if

$$
\mathcal{L}[y(t) ; h]=\mathcal{O}\left(h^{p+1}\right),
$$

for all $y(t)$ sufficiently smooth.

Note that the preconsistency condition $A \mathbf{e}=\mathbf{e}$ is equivalent to $\mathcal{L}[1 ; h]=\mathbf{0}$ and the method has order $p$ and error constant $\mathbf{C}_{p+1}$ iff the $s$-dim vector linear operator $\mathcal{L}$ defined by (12) satisfies

$$
\mathcal{L}\left[t^{j} ; h\right]=\mathbf{0}, \quad \text { for } \quad j=1, \ldots, p \quad \text { and } \quad \mathcal{L}\left[t^{p+1} ; h\right]=\mathbf{C}_{p+1}(p+1) ! h^{p+1} \neq \mathbf{0} .
$$

Remark 1. Since $\mathcal{L}$ defined by (12) is a linear operator of $y(t)$ the Taylor series expansion at $t$ will have the form

$$
\mathcal{L}[y(t) ; h]=\mathbf{C}_{0} y(t)+\mathbf{C}_{1} y^{\prime}(t) h^{2}+\mathbf{C}_{2} y^{\prime \prime}(t) h^{2}+\ldots
$$

with some $s$-valued constants $\mathbf{C}_{j}=\mathbf{C}_{j}\left(A, B, R, c_{1}, \ldots, c_{s}\right)$ and if the method has order $p$ then $\mathbf{C}_{j}=\mathbf{0}, j=0,1, \ldots, p$ and $\mathbf{C}_{p+1} \neq \mathbf{0}$. Because of this linear dependence on $y(t)$, both the order and the error constant $\mathbf{C}_{p+1}$ are independent of the time $t$ of expansion. This implies that by choosing e.g. the time expansion at $t^{*}=t+c_{1} h$, since $\mathbf{Y}(t)=\left(y\left(t^{*}\right), y\left(t^{*}+\left(c_{2}-c_{1}\right) h\right), \ldots, y\left(t^{*}+\left(c_{s}-c_{1}\right) h\right)\right)^{T}$ depends only on the differences $c_{j}-c_{1}$, the coefficients of the Taylor's expansion

$$
\mathcal{L}\left[y\left(t^{*}\right) ; h\right]=\mathbf{D}_{0} y\left(t^{*}\right)+\mathbf{D}_{1} y^{\prime}\left(t^{*}\right) h^{2}+\mathbf{D}_{2} y^{\prime \prime}\left(t^{*}\right) h^{2}+\ldots
$$

$\mathbf{D}_{j}=\mathbf{D}_{j}\left(A, B, R, c_{2}-c_{1}, \ldots, c_{s}-c_{1}\right)$ depend only on the relative differences to the first node $c_{1}$, and similarly for any other node. Thus, by fixing a given node $c_{k}$, the order conditions as well as the error constant depend only on $c_{j}-c_{k}, j \neq k$.

Putting $\widehat{\mathcal{L}}[y(t) ; h]=P \mathcal{L}[y(t) ; h]$, in view of $(12)$ it can be written as

$$
\widehat{\mathcal{L}}[y(t) ; h]=P \mathbf{Y}(t+h)-\widehat{A} P \mathbf{Y}(t)-h \widehat{B} P \mathbf{Y}^{\prime}(t)-h \widehat{R} P \mathbf{Y}^{\prime}(t+h),
$$

and because of $(10)$, condition (13) is equivalent to $\widehat{\mathcal{L}}[y(t) ; h]=\mathcal{O}\left(h^{p+1}\right)$. 
Denoting by $\mathbf{Z}(t)=\left(z_{j}(t)\right)_{j=1}^{s} \equiv P \mathbf{Y}(t) \equiv P y(t \mathbf{e}+h \mathbf{c})$, the linear operator $\widehat{\mathcal{L}}[y(t) ; h]$ of $(14)$ can be written equivalently as

$$
\widehat{\mathcal{L}}[y(t) ; h]=\mathbf{Z}(t+h)-\widehat{A} \mathbf{Z}(t)-h \widehat{B} \mathbf{Z}^{\prime}(t)-h \widehat{R} \mathbf{Z}^{\prime}(t+h),
$$

and the method has order $p$ if the right hand side of $(15)$ is $\mathcal{O}\left(h^{p+1}\right)$.

To introduce a further simplification in the study of the order conditions, we define a new scaled time $\tau$ so that $t=\tau h$ and a new function $u(\tau)=y(h \tau)$. Then $\mathbf{Z}(t)=P y(t \mathbf{e}+h \mathbf{c})=P u(\tau \mathbf{e}+\mathbf{c})$, and putting $\xi[u(\tau)]=P u(\tau \mathbf{e}+\mathbf{c}) \in \mathbb{R}^{s}$, the method (6),(8),(9) has order $p$ iff

$$
\widehat{A} \xi[u(\tau)]+\widehat{B} \dot{\xi}[u(\tau)]+\widehat{R} \dot{\xi}[u(\tau+1)]=\xi[u(\tau+1)],
$$

holds for all $u(\tau) \in \Pi_{p}(\tau)$ (the set of polynomials in $\tau$ with degree $\leq p$ ), where the dot denotes the derivative with respect to $\tau$. Further by the linear dependence of $\xi$ on $u(\tau)$, it is enough to check (16) for $\tau=0$.

Next we will show that, for a given admissible set of nodes, under some minor restrictions on the components of $P$ the equations of (16) possess a unique solution in the free parameters of $\widehat{A}, \widehat{B}$ and $\widehat{R}$ with stage order $2 s-1$. As a first step to establish the existence of $s$-stage methods with stage order $2 s-1$ we prove the following

Lemma 1. For a given admissible set of nodes $c_{i}, i=1, \ldots, s$ and arbitrary constants $p_{i j}, 1 \leq j<i \leq s$, consider the construction of a method of order $p=2 s-1$.

- The first stage order equation of (16) possesses a unique solution in the unknowns of the first row of (11) i.e.

$$
\widehat{a}_{12}, \ldots, \widehat{a}_{1 s}, \widehat{b}_{11}, \ldots, \widehat{b}_{1 s} .
$$

- The last stage order equation of (16) possesses a unique solution in the unknowns of the last row of (11) i.e.

$$
\widehat{b}_{s 1}, \ldots, \widehat{b}_{s s}, \widehat{r}_{s 1}, \ldots, \widehat{r}_{s, s-1} \text {. }
$$

Proof. Since the first stage order equation is a linear equation in the unknowns (17) it will be enough to show that the corresponding homogeneous equation of (16) possesses only the trivial solution, i.e., that if for any polynomial $u(\tau) \in$ $\Pi_{2 s-1}(\tau)$ we have

$$
\sum_{j=2}^{s} \widehat{a}_{1 j} \xi_{j}[u(0)]+\sum_{j=1}^{s} \widehat{b}_{1 j} \dot{\xi}_{j}[u(0)]=0,
$$

then $\widehat{a}_{1 j}=0, j=2, \ldots, s$ and $\widehat{b}_{1 j}=0, j=1, \ldots, s$. 
By choosing for $u(\tau)$ the polynomial

$$
u(\tau)=\frac{\left(\tau-c_{1}\right)^{2}\left(\tau-c_{2}\right)^{2} \ldots\left(\tau-c_{s-1}\right)^{2}\left(\tau-c_{s}\right)}{\left(c_{s}-c_{1}\right)^{2}\left(c_{s}-c_{2}\right)^{2} \ldots\left(c_{s}-c_{s-1}\right)^{2}} \in \Pi_{2 s-1}(\tau),
$$

we have

$$
u\left(c_{j}\right)=0,(j=1, \ldots, s), \dot{u}\left(c_{j}\right)=0,(j=1, \ldots, s-1), \dot{u}\left(c_{s}\right)=1,
$$

and therefore

$$
\xi_{j}[u(0)]=\dot{\xi}_{j}[u(0)]=0,(j=1, \ldots, s-1), \xi_{s}[u(0)]=0, \dot{\xi}_{s}[u(0)]=1,
$$

and by substituting into (19) we get $\widehat{b}_{1 s}=0$.

Next repeating the process with the polynomial

$$
u(\tau)=\frac{\left(\tau-c_{1}\right)^{2}\left(\tau-c_{2}\right)^{2} \ldots\left(\tau-c_{s-2}\right)^{2}\left(\tau-c_{s-1}\right)\left(\tau-c_{s}\right)}{\left(c_{s-1}-c_{1}\right)^{2}\left(c_{s-1}-c_{2}\right)^{2} \ldots\left(c_{s-1}-c_{s-2}\right)^{2}\left(c_{s-1}-c_{s}\right)}
$$

we arrive to $\widehat{b}_{1 s-1}=0$. And following in this way we obtain that all $\widehat{b}_{1 j}=0$.

After that, with the polynomials

$$
u_{k}(\tau)=\prod_{j=1, j \neq k}^{s} \frac{\tau-c_{j}}{c_{k}-c_{j}} \in \Pi_{s-1}(\tau), \quad k=s, s-1, \ldots, 1
$$

we derive successively the vanishing of the coefficients $\widehat{a}_{1 s}, \widehat{a}_{1, s-1}, \ldots, \widehat{a}_{11}$ of $(19)$.

The proof for the last stage order equation follows a similar pattern. We need to show that if

$$
\sum_{j=1}^{s} \widehat{b}_{s j} \dot{\xi}_{j}[u(0)]+\sum_{j=1}^{s-1} \widehat{r}_{s j} \dot{\xi}_{j}[u(1)]=0
$$

holds for all polynomials $u(\tau)$ of degree $\leq 2 s-1$ then all $\widehat{b}_{s j}$ and $\widehat{r}_{s j}$ must be zeroes.

Taking a polynomial $u(\tau)=\int_{0}^{\tau} v(\tau) \in \Pi_{2 s-1}(\tau)$ with (remark that we consider an admissible set $\left.\left\{c_{i} \mid i=1, \ldots, s\right\}\right)$

$$
v(\tau)=\frac{\left(\tau-c_{1}\right) \ldots\left(\tau-c_{s}\right)\left(\tau-\left(c_{1}+1\right)\right) \ldots\left(\tau-\left(c_{s-2}+1\right)\right)}{\left(c_{s-1}-c_{1}\right) \ldots\left(c_{s-1}-c_{s}\right)\left(c_{s-1}-\left(c_{1}+1\right)\right) \ldots\left(c_{s-1}-\left(c_{s-2}+1\right)\right)},
$$

we obtain that $\widehat{r}_{s, s-1}=0$. In this way we derive successively $\widehat{r}_{s, s-2}=0, \ldots, \widehat{r}_{s 1}=$ 0 and then $\widehat{b}_{s j}=0, j=s, \ldots 1$.

For the order equations corresponding to the stages $k=2, \ldots, s-1$ the existence of a unique solution of the $k$-th component of the vector equation (16) in the unknowns $\widehat{a}_{k, k+1}, \ldots, \widehat{a}_{k s}, \widehat{b}_{k 1}, \ldots, \widehat{b}_{k s}, \widehat{r}_{k 1}, \ldots, \widehat{r}_{k, k-1}$ requires an additional 
relation between the nodes and the elements of $P$ at each stage. In fact, the $k$-th stage order equation of (16) is given by

$$
\sum_{j=k+1}^{s} \widehat{a}_{k j} \xi_{j}[u(0)]+\sum_{j=1}^{s} \widehat{b}_{k j} \dot{\xi}_{j}[u(0)]+\sum_{j=1}^{k-1} \widehat{r}_{k j} \dot{\xi}_{j}[u(1)]=\xi_{k}[u(1)],
$$

where $u(\tau) \in \Pi_{2 s-1}(\tau)$. Note that for $u(\tau)=1, \xi[u(\tau)]=P \mathbf{e}=\mathbf{e}_{1}$ and $\dot{\xi}[u(\tau)]=P \mathbf{0}=\mathbf{0}$ and then (21) is clearly satisfied. Hence it is enough to require (21) for the remaining elements of a basis of $\Pi_{2 s-1}(\tau)$. Taking $u(\tau)=$ $\tau^{m}, m=1, \ldots, 2 s-1$ this condition becomes

$$
\operatorname{det}\left[\mu_{k+1}(0), \ldots, \mu_{s}(0), \dot{\mu}_{1}(0), \ldots, \dot{\mu}_{s}(0), \dot{\mu}_{1}(1), \ldots, \dot{\mu}_{k-1}(1)\right] \neq 0
$$

with

$$
\mu_{j}(\tau)=\left(\sum_{i=1}^{j} p_{j i}\left(\tau+c_{i}\right), \ldots, \sum_{i=1}^{j} p_{j i}\left(\tau+c_{i}\right)^{2 s-1}\right)^{T} \in \mathbb{R}^{2 s-1} .
$$

Condition (22) can be expressed in a simpler way in terms of the determinant of a matrix with dimension $s-k-1$. Let us consider for example the order conditions of the second stage $(k=2)$ for the case of four stages $(s=4)$. According to (21) there will be a unique solution if and only if

$$
\sum_{j=3}^{4} \widehat{a}_{2 j}\left(\sum_{l=1}^{j} p_{j l} u\left(c_{l}\right)\right)+\sum_{j=1}^{4} \widehat{b}_{2 j}\left(\sum_{l=1}^{j} p_{j l} \dot{u}\left(c_{l}\right)\right)+\widehat{r}_{21} \dot{u}\left(1+c_{1}\right)=0,
$$

for all $u(\tau) \in \Pi_{7}(\tau)$ implies that all involved $\widehat{a}_{2 j}, \widehat{b}_{2 j}$ and $\widehat{r}_{21}$ coefficients are zero.

Taking the polynomials $u_{6}(\tau) \in \Pi_{6}(\tau)=\Pi_{2 s-2}(\tau)$ and $u_{7}(\tau) \in \Pi_{7}(\tau)=$ $\Pi_{2 s-1}(\tau)$ such that

$$
\dot{u}_{6}(\tau) \equiv \Pi_{i=1}^{5}\left(\tau-c_{i}\right), \quad \dot{u}_{7}(\tau) \equiv \dot{u}_{6}(\tau) \tau,
$$

with $c_{5}=c_{1}+1$, clearly the coefficients of $\widehat{b}_{2 j}$ and $\widehat{r}_{21}$ in (24) vanish and we have the two linear equations

$$
\sum_{j=3}^{4} \widehat{a}_{2 j}\left(\sum_{l=1}^{j} p_{j l} u_{m}\left(c_{l}\right)\right)=0, \quad m=6,7
$$

and, due to the consistency condition $P \mathbf{e}=\mathbf{e}_{1}$,

$$
\widehat{a}_{23}\left(\sum_{j=1}^{3} p_{3 j}\left(u_{m}\left(c_{j}\right)-u_{m}(0)\right)\right)+\widehat{a}_{24}\left(\sum_{j=1}^{4} p_{4 j}\left(u_{m}\left(c_{j}\right)-u_{m}(0)\right)\right)=0,
$$

for $m=6$ and $m=7$. Suppose we rewrite both equations as

$$
\widehat{a}_{23} \alpha_{6}+\widehat{a}_{24} \beta_{6}=0, \quad \widehat{a}_{23} \alpha_{7}+\widehat{a}_{24} \beta_{7}=0,
$$


then it is clear that this interpolation problem has a unique solution unless its coefficient matrix is singular, that is, when $\alpha_{6} \beta_{7}=\alpha_{7} \beta_{6}$, which gives us a condition on coefficients $p_{i j}$ and nodes $c_{i}$.

In general, for stage $k$ with $1<k<s$, let $P^{(k)}$ be the $(s-k) \times s$ matrix that is obtained from $P$ after deleting the first $k$ rows, and let $Q^{(k)}$ be the $s \times(s-k)$ matrix with

$$
Q_{i j}^{(k)}=\int_{0}^{c_{i}} \prod_{q=1}^{s+k-1}\left(x-c_{q}\right) x^{j-1} \mathrm{~d} x,
$$

and $c_{s+j}=1+c_{j}$. Then in order to have a unique solution, the square matrices $P^{(k)} Q^{(k)}$ should be non-singular, that is

$$
\operatorname{det}\left[P^{(k)} Q^{(k)}\right] \neq 0 \text {, for all } k=2, \ldots, s-1 \text {. }
$$

To prove that there is a unique solution for the $\widehat{b}_{k q}$ and $\widehat{r}_{k q}$ coefficients of stage equation $k$, we can follow the same procedure as the one used for the last stage. We may therefore state the following

Theorem 2. For all $s \geq 2$ there is a family of s-stage, fixed stepsize, two step peer methods (6),(8),(9) with order $2 s-1$ that depends on the $s+(s-2)(s-1) / 2$ parameters (the $s$ non confluent nodes $c_{i}$ and the $(s-2)(s-1) / 2$ elements of $P$ with $P \mathbf{e}=\mathbf{e}_{1}$ ) with the $s-2$ relations (26). The coefficients $\widehat{A}, \widehat{B}, \widehat{R}$ are uniquely determined by (16) for $u(\tau) \in \Pi_{2 s-1}(\tau)$.

Remark 2. According to Remark 1, for a method with $s$ stages and order $2 s-1$, the available parameters in $\widehat{A}, \widehat{B}, \widehat{R}$ depend only on the $s-1$ differences $c_{k}-c_{l}, k \neq l$ to a fixed node $c_{l}$. This allows us to choose a pivot node. Assuming $c_{1}<\ldots<c_{s}$, the choice $c_{s}=1$ (used for example in [18]) provides the numerical solution at grid points but, if some $c_{j}<0$, the method uses information of the back step and requires a special starting procedure. On the other hand, if $c_{1}=0$ and some $c_{j}>1$, the final step must be taken so that the last stage gives the approximation to the solution at the end point of the integration interval.

In the case of two-step methods with variable stepsize, let us suppose that we advance the numerical solution from the known stages $Y_{0, j}$ corresponding to the points $t_{0, j}=t_{0}+c_{j} h$, obtained with stepsize $h$ and we want to modify the size of the next step by a factor $\sigma$, so that the new $Y_{1, k} \simeq y\left(t_{1}+\sigma h\right)=y\left(t_{0}+h+\sigma h\right)$ are given by

$$
Y_{1, j}=\sum_{k=1}^{s} a_{j k} Y_{0, k}+h \sum_{k=1}^{s} b_{j k} f_{0, k}+\sigma h \sum_{k=1}^{j-1} r_{j k} f_{1, k}
$$

With the notations (4), for a scalar case they can be written in the vector form

$$
\mathbf{Y}_{1}=A \mathbf{Y}_{0}+h B f\left(t_{0} \mathbf{e}+h \mathbf{c}, \mathbf{Y}_{0}\right)+(\sigma h) R f\left(t_{1} \mathbf{e}+\sigma h \mathbf{c}, \mathbf{Y}_{1}\right)
$$

where now the matrices $A(\sigma), B(\sigma)$ and $R(\sigma)$ can depend on the stepsize ratio $\sigma$. 
To ensure the zero-stability, we are considering methods with

$$
A=P^{-1} \widehat{A} P
$$

with $P$ constant, lower triangular, satisfying the condition $P \mathbf{e}=\mathbf{e}_{1}$ and $\widehat{A}=$ $\widehat{A}(\sigma)$ as in (9) so that $A$ has the eigenvalue unity (simple) and the remaining eigenvalues are zero.

It is well known that the zero-stability is guaranteed if any product of consecutive matrices

$$
A\left(\sigma_{k+r}\right) A\left(\sigma_{k+r-1}\right) \ldots A\left(\sigma_{r}\right),
$$

is uniformly bounded for all $k, r$. Note that the stability depends on the sequence of step sizes $\left\{h_{0}, h_{1}, \ldots\right\}$. Weiner and co-workers choose the matrix $A$ constant with all the eigenvalues equal to zero except one, simple, equal to one. In this way, the method is zero-stable for any sequence of step sizes $\left\{h_{j}\right\}$. In our case, since $P$ is constant, the stability is equivalent to the uniform boundedness of the product of matrices

$$
\left\|\widehat{A}\left(\sigma_{k+r}\right) \widehat{A}\left(\sigma_{k+r-1}\right) \ldots \widehat{A}\left(\sigma_{r}\right)\right\| \leq K<\infty, \text { for all } k, r,
$$

and this is ensured if the coefficients $\widehat{a}_{i j}$ (that can depend on the step size ratios $\left.\sigma_{n}\right)$ are uniformly bounded.

Theorem 3. The variable stepsize two-step peer method (27) with matrix A satisfying (8), (9) and $\widehat{A}=\widehat{A}(\sigma)$ is zero-stable for any step size sequence such that the coefficients of the matrix $\widehat{A}$ are uniformly bounded.

Proof. To prove (29) we will see that for all fixed $r$ and $k \geq s-2$

$$
\widehat{A}\left(\sigma_{r+k}\right) \widehat{A}\left(\sigma_{r+k-1}\right) \ldots \widehat{A}\left(\sigma_{r}\right)=\widehat{A}\left(\sigma_{r+s-2}\right) \widehat{A}\left(\sigma_{r+s-3}\right) \ldots \widehat{A}\left(\sigma_{r}\right) .
$$

Decomposing the matrix $\widehat{A}=\widehat{A}(\sigma)$ in blocks

$$
\widehat{A}(\sigma)=\left(\begin{array}{l|l}
1 & d(\sigma)^{T} \\
\hline 0 & T(\sigma)
\end{array}\right),
$$

and taking into account that $T(\sigma)$ is strictly upper triangular matrix of dimension $s-1$, it is easy to see that

$$
\widehat{A}\left(\sigma_{r+s-2}\right) \widehat{A}\left(\sigma_{r+s-3}\right) \ldots \widehat{A}\left(\sigma_{r}\right)=\left(\begin{array}{c|c}
1 & d_{s, r}^{T} \\
\hline 0 & 0
\end{array}\right),
$$

with

$$
d_{s, r}^{T}=d\left(\sigma_{r}\right)^{T}+d\left(\sigma_{r+1}\right)^{T} T\left(\sigma_{r}\right)+\ldots+d\left(\sigma_{r+s-2}\right)^{T} T\left(\sigma_{r+s-3}\right) \ldots T\left(\sigma_{r}\right) .
$$

Consequently, the product of $s$ consecutive matrices is a matrix with only the first row non zero, and with the first element of this row unity. From here, it is clear that (29) holds. 
Then, if $\widehat{a}_{i j}\left(\sigma_{n}\right)$ are uniformly bounded for the $\sigma_{n}$ ratios, the matrices $\widehat{A}\left(\sigma_{n}\right)$ are also uniformly bounded, $\left\|\widehat{A}\left(\sigma_{n}\right)\right\| \leq K$, where $K \geq 1$ since $A \mathbf{e}=\mathbf{e}$. Therefore, for any product of consecutive matrices we have the bound

$$
\left\|\widehat{A}\left(\sigma_{r+k}\right) \widehat{A}\left(\sigma_{r+k-1}\right) \ldots \widehat{A}\left(\sigma_{r}\right)\right\| \leq K^{\min \{k+1, s-1\}} \leq K^{s-1} .
$$

Let us remark that with the above choice of the transition matrix $A=$ $A(\sigma)=P^{-1} \widehat{A}(\sigma) P$ the calculation of coefficients for variable step size is very similar to the case of fixed step size. To find the expressions for the coefficients of the matrices, we only have to take into account that the interpolation points are now given by $\left\{c_{1}, c_{2} \ldots, c_{s}, 1+\sigma_{n} c_{1}, \ldots, 1+\sigma_{n} c_{s}\right\}$ and that $R$ is replaced by $\sigma_{n} R$. Note that now, we can not ensure that $c_{i} \neq 1+\sigma c_{j}$ for all values of $\sigma$, unless $c_{i} \in(0,1]$ or $c_{i} \in[0,1)$ for all $i$.

By introducing the linear operator corresponding to (15)

$$
\begin{aligned}
\widehat{\mathcal{L}}[y(t), h, \sigma] \equiv & P \mathbf{Y}(t+h, \sigma h)-\widehat{A} P \mathbf{Y}(t, h) \\
& -h \widehat{B} P \mathbf{Y}^{\prime}(t, h)-(\sigma h) \widehat{R} P \mathbf{Y}^{\prime}(t+h, \sigma h),
\end{aligned}
$$

where now the two-arguments vector function $\mathbf{Y}\left(t_{1}, h_{1}\right)$ is defined by

$$
\mathbf{Y}\left(t_{1}, h_{1}\right)=y\left(t_{1} \mathbf{e}+h_{1} \mathbf{c}\right)=\left(y\left(t_{1}+c_{j} h_{1}\right)\right)_{j=1}^{s} \in \mathbb{R}^{s},
$$

the method (27) has order $p$ iff the operator $\widehat{\mathcal{L}}[y(t), h, \sigma]$ defined by $(31),(32)$ satisfies $\widehat{\mathcal{L}}[y(t), h, \sigma]=\mathcal{O}\left(h^{p+1}\right)$, uniformly for $\sigma$ in some compact $J_{\sigma}$ containing $\sigma=1$, or equivalently iff $\widehat{\mathcal{L}}[y(t), h, \sigma]=0, \forall y(t) \in \Pi_{p}(t)$.

To simplify the derivation of the order conditions we introduce again a new time $\tau$ by $t=h \tau$ and $u(\tau)=y(t)=y(h \tau)$. Then defining the $s$-valued vector functions

$$
\xi[u](\tau, \sigma)=\left(\xi_{j}[u](\tau, \sigma)\right)_{j=1}^{s}=P u(\tau \mathbf{e}+\sigma \mathbf{c}) .
$$

The variable stepsize method (27) has order $p$ if and only if

$$
\widehat{A} \xi[u](\tau, 1)+\widehat{B} \dot{\xi}[u](\tau, 1)+\sigma \widehat{R} \dot{\xi}[u](\tau, \sigma)=\xi[u](\tau, \sigma)
$$

holds for all polynomial $u(\tau) \in \Pi_{p}(\tau)$, where dots denote derivative with respect to $\tau$.

The point we want to address now is under which conditions on the stepsize ratios $\sigma_{n}$ a method obtained with fixed stepsize and order $p$ can be extended to a zero-stable, $p$ th order, variable step size method, according to equations (33).

Let us suppose that for some fixed admissible nodes $c_{i}$ and matrix $P$ we have a fixed stepsize method of order $p=2 s-1$. Equations (33) are continuous on $\sigma$ and have solution for $\sigma=1$, therefore they define a unique solution $A(\sigma), B(\sigma), R(\sigma)$ for some neighborhood around $\sigma=1$. Moreover, since for $u(\tau) \in \Pi_{p}(\tau)$ the equations (33) are polynomic on $\sigma$, the coefficients of the solution matrices $\widehat{A}(\sigma), \widehat{B}(\sigma), \widehat{R}(\sigma)$ will be rational on $\sigma$. Hence, the coefficients of the variable stepsize method will be defined for all $\sigma$ except for at most a 
finite number of values of the stepsize ratio $\sigma$. Let us suppose, without loss of generality, that there is only one such singular value of $\sigma, \sigma_{1}^{*}$. For any small value $\varepsilon_{1}>0$, the method with fixed stepsize can be extended to variable stepsize for all $\sigma \in J_{\sigma}=\left[0, \sigma_{\max }\right] \backslash\left(\sigma_{1}^{*}-\varepsilon_{1}, \sigma_{1}^{*}+\varepsilon_{1}\right)$. In practice, if a stepsize ratio $\sigma \in\left(\sigma_{1}^{*}-\varepsilon_{1}, \sigma_{1}^{*}+\varepsilon_{1}\right)$ is selected by the stepsize control algorithm, the value $\sigma=\sigma_{1}^{*}-\varepsilon_{1}$ can be taken instead to guarantee the existence of the method yet maintaining the local truncation error under the prescribed error tolerance. The zero-stability is guaranteed because $J_{\sigma}$ is a compact set and the coefficients of $A(\sigma)$ are continuous.

Note that the construction of suitable peer methods will require the selection of the free parameters $c_{i}$ and $p_{i j}$ such that the method has maximum order (for example $p=2 s-1$ ), maximum stability domain and minimum forbidden stepsize ratios $\sigma_{1}^{*}, \sigma_{2}^{*}, \ldots$ In addition, for the valid stepsize ratios $\sigma$ the corresponding coefficients of the matrices $A(\sigma), B(\sigma), R(\sigma)$ should not be too large, and $\varepsilon_{1}, \varepsilon_{2}, \ldots$ should be as small as possible.

\section{Peer methods with $s=2$}

From the previous section, it follows that a two-stage peer method of order three can be obtained for any choice of $c_{1}$ and $c_{2}$ for which $\left(c_{1}-c_{2}\right)\left(1-c_{1}-c_{2}\right) \neq$ 0 . Indeed, solving the order conditions (16) leads to :

$$
\widehat{A}=\left(\begin{array}{cc}
1 & \frac{-2+3 d}{d^{3}} \\
0 & 0
\end{array}\right), \widehat{B}=\left(\begin{array}{cc}
\frac{2-3 d+d^{2}}{d^{2}} & \frac{1-d}{d^{2}} \\
\frac{(6-d) d^{2}}{6(d-1)} & \frac{d(3+2 d)}{6(d-1)}
\end{array}\right), \widehat{R}=\left(\begin{array}{cc}
0 & 0 \\
\frac{d\left(d^{2}-6\right)}{6(d-1)} & 0
\end{array}\right)
$$

where $0 \neq d \neq 1$ with $d:=c_{2}-c_{1}$. The leading term in the Taylor's expansion of $\widehat{\mathcal{L}}[y(t), h]$ is given by

$$
\left(\begin{array}{c}
\frac{1}{24}(d-1)^{2} \\
-\frac{1}{72}\left(d^{2}-2 d-6\right) d^{2}
\end{array}\right) h^{4} y^{(4)}(t)
$$

so that no method with $s=2$ can be found of order 4 . It is even impossible to impose that one stage has order 4 , while the other remains of order 3.

The matrix $P$, given in $(9)$, reads $P=\left(\begin{array}{cc}1 & 0 \\ -1 & 1\end{array}\right)$, which means that

$$
A=\left(\begin{array}{cc}
1-\widehat{a}_{12} & \widehat{a}_{12} \\
1-\widehat{a}_{12} & \widehat{a}_{12}
\end{array}\right), \quad B=\left(\begin{array}{cc}
\widehat{b}_{11}-\widehat{b}_{12} & \widehat{b}_{12} \\
\widehat{b}_{11}+\widehat{b}_{21}-\widehat{b}_{12}-\widehat{b}_{22} & \widehat{b}_{12}+\widehat{b}_{22}
\end{array}\right), \quad R=\widehat{R}
$$

and, since $\mathcal{L}[y(t), h]=P^{-1} \widehat{\mathcal{L}}[y(t), h]$, its leading term is

$$
\left(\begin{array}{c}
\frac{1}{24}(d-1)^{2} \\
\frac{1}{72}\left(3-6 d+9 d^{2}+2 d^{3}-d^{4}\right)
\end{array}\right) h^{4} y^{(4)}(t)
$$


The characteristic equation of $M(z)$ is given by

$$
p_{2}(\lambda)=\operatorname{det}\left(M_{2}(z, d)-\lambda I\right)=\lambda^{2}-T \lambda+D,
$$

whereby

$$
T=1+z \frac{d^{2}+7 d-6}{3 d}+z^{2} \frac{6-d^{2}}{6 d}, \quad D=z \frac{d^{2}+4 d-6}{3 d}+z^{2} \frac{d^{2}+5 d-6}{6 d} .
$$

Given a value for $d$, stability along the imaginary axis is then obtained for those values of $z$ for which the spectral radius $\rho(M)<1$. This study can be carried out by means of the Routh-Hurwitz criterion [5, pp. 194], that allows to check whether or not all the roots of a polynomial $q(\mu)$ with real coefficients have a negative real part. Before doing so, it is necessary to use the mapping $\lambda \rightarrow \mu$ defined by

$$
\mu=\frac{\lambda+1}{\lambda-1},
$$

which transforms the interior of the unit disk of the $\lambda$-plane in the left half of the $\mu$-plane. This leads to

$$
q_{2}(\mu)=(1-\mu)^{2} p_{2}(\lambda)=E_{1}+E_{2} \mu+E_{3} \mu^{2},
$$

whereby

$$
\begin{aligned}
& E_{1}=1+T+D=2+\frac{-12+11 d+2 d^{2}}{3 d} z+\frac{5}{6} z^{2}=2+\delta z+\frac{5}{6} z^{2}, \\
& E_{2}=2+\frac{12-8 d-2 d^{2}}{3 d} z+\frac{6-5 d-d^{2}}{3 d} z^{2}=2+\gamma_{1} z+\gamma_{2} z^{2}, \\
& E_{3}=1-T+D=-z+\frac{2 d^{2}+5 d-12}{6 d} z^{2} .
\end{aligned}
$$

The Routh-Hurwitz criterion shows that the roots will belong to $\mathbb{C}^{-}$iff $E_{1}$, $E_{2}$ and $E_{3}$ have the same sign. A detailed analysis gives the following result, shown in the left part in Figure 1 : the stability interval along the negative real axis as a function of $d \in[-2,2]$ is given by $[\rho, 0]$ whereby

$$
\rho= \begin{cases}\frac{-3 d-\sqrt{9 d^{2}-60}}{5} & \text { if }-2 \leq d<0, \\ \frac{-6 d}{12-5 d-2 d^{2}} & \text { if } d \in\left(0, d_{\max }\right], \\ \frac{-\gamma_{1}+\sqrt{\gamma_{1}^{2}-8 \gamma_{2}}}{2 \gamma_{2}} & \text { if } d \in\left[d_{\max }, d_{\mathrm{disc}}\right), \\ \frac{-3 \delta+\sqrt{9 \delta^{2}-60}}{5} & \text { if } d_{\mathrm{disc}} \leq d \leq 2,\end{cases}
$$



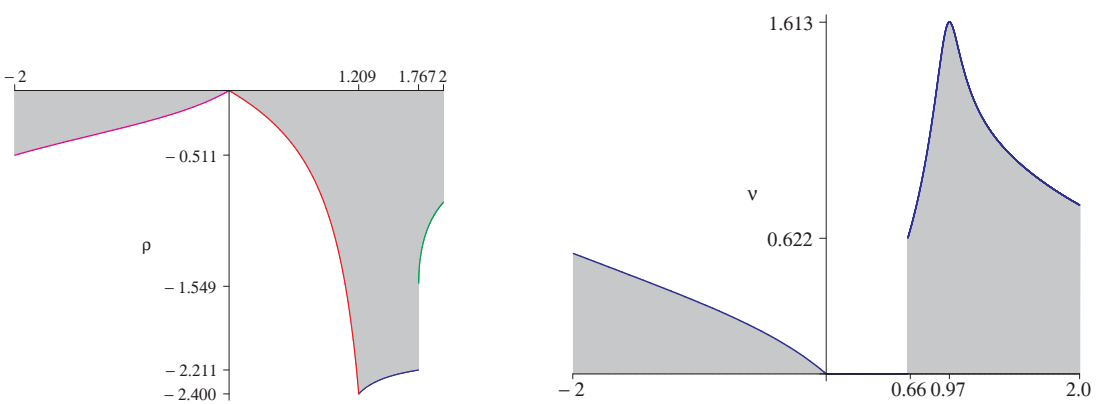

Figure 1: Stability along the negative real axis (left) and the positive imaginary axis (right) for the two-stage peer method as a function of $d$.

where $d_{\max }=(-15+\sqrt{609}) / 8=1.20974 \ldots$ and $d_{\text {disc }}=1.76753 \ldots$ is a root of $9 \delta^{2}-60=0$. The optimal value for $d$, considering only stability along the real axis, is thus given by $d_{\max }$.

Let us now consider stability along the imaginary axis. Since the RouthHurwitz criterion can not be used, we can only rely on a numerical procedure, which resulted in the right part of Figure 1. There we show $\nu$ as a function of $d$, where $[-\nu, \nu]$ is the interval of stability along the imaginary axis. For small positive values of $d$, this interval is empty but the stability region is in fact very close to the imaginary axis. From $d \approx 0.663$ onward, the interval is non-empty and a maximum is reached at $d=0.972 \ldots$.

Considering both stability along the real axis and along the imaginary axis, it is clear that the optimal values are obtained for $d=d_{\max }$, for which $\rho=-2.4$ and $\nu=1.199 \ldots$ 
For this value $d=d_{\max }$ the coefficients of the method are $c_{2}=c_{1}+d_{\mathrm{disc}}$,

$$
\begin{aligned}
& A=\left(\begin{array}{ll}
\frac{2169-73 \sqrt{609}}{4608} & \frac{2439+73 \sqrt{609}}{4608} \\
\frac{2169-73 \sqrt{609}}{4608} & \frac{2439+73 \sqrt{609}}{4608}
\end{array}\right)=\left(\begin{array}{ll}
0.0797551 & 0.920245 \\
0.0797551 & 0.920245
\end{array}\right), \\
& B=\left(\begin{array}{ll}
\frac{283-11 \sqrt{609}}{384} & \frac{19-3 \sqrt{609}}{384} \\
\frac{-911+43 \sqrt{609}}{384} & \frac{835+45 \sqrt{609}}{384}
\end{array}\right)=\left(\begin{array}{ll}
0.0300594 & -0.143317 \\
0.391018 & 5.06642
\end{array}\right), \\
& R=\left(\begin{array}{cc}
0 & 0 \\
\frac{-57-9 \sqrt{609}}{64} & 0
\end{array}\right)=\left(\begin{array}{rr}
0 & 0 \\
-4.36096 & 0
\end{array}\right),
\end{aligned}
$$

and the leading term of the local truncation error is

$$
\frac{1}{4 !}\left(\begin{array}{c}
\frac{569}{32}-\frac{23}{32} \sqrt{609} \\
-\frac{49891}{512}+\frac{2093}{512} \sqrt{609}
\end{array}\right) h^{4} y^{(4)}\left(t_{0}\right)=\left(\begin{array}{c}
0.001833 \ldots \\
0.143221 \ldots
\end{array}\right) h^{4} y^{(4)}\left(t_{0}\right)
$$

such that $\left\|\mathbf{C}_{4}\right\|_{2}=0.143236$.

Let us analyze now the extension of the method obtained to variable stepsize. The expressions for the non-zero coefficients of the matrices $\widehat{A}(\sigma), \widehat{B}(\sigma)$ and $\widehat{R}(\sigma)$ can be written down in terms of (only differences between) $c_{1}, c_{2}=c_{1}+d$, $c_{3}=1+\sigma c_{1}$ and $c_{4}=1+\sigma c_{2}$. One then obtains

$$
\begin{aligned}
\widehat{a}_{12} & =\frac{\left(c_{1}-c_{3}\right)^{2}\left(c_{1}-3 c_{2}+2 c_{3}\right)}{\left(c_{1}-c_{2}\right)^{3}} \\
\widehat{b}_{11} & =-\frac{\left(c_{2}-c_{3}\right)\left(c_{1}-c_{3}\right)\left(c_{1}+c_{2}-2 c_{3}\right)}{\left(c_{1}-c_{2}\right)^{2}} \\
\widehat{b}_{12} & =-\frac{\left(c_{1}-c_{3}\right)^{2}\left(c_{2}-c_{3}\right)}{\left(c_{1}-c_{2}\right)^{2}} \\
\widehat{b}_{21} & =\frac{1}{6} \frac{\left(c_{3}-c_{4}\right)^{2}\left(-4 c_{3}-2 c_{4}+3 c_{2}+3 c_{1}\right)}{\left(c_{2}-c_{3}\right)\left(c_{1}-c_{3}\right)} \\
\widehat{b}_{22} & =\frac{1}{6} \frac{\left(c_{3}-c_{4}\right)^{2}\left(-c_{3}+3 c_{1}-2 c_{4}\right)}{\left(c_{2}-c_{3}\right)\left(c_{1}-c_{2}\right)} \\
\widehat{r}_{21} & =-\frac{1}{6} \frac{\left(c_{3}-c_{4}\right)\left(2 c_{3}^{2}+2 c_{3} c_{4}-3 c_{2} c_{3}-3 c_{1} c_{3}+6 c_{1} c_{2}+2 c_{4}^{2}-3 c_{2} c_{4}-3 c_{1} c_{4}\right)}{\sigma\left(c_{2}-c_{3}\right)\left(c_{1}-c_{3}\right)}
\end{aligned}
$$


This holds for all $c_{1}, c_{2}, c_{3}$ such that $\left(c_{1}-c_{2}\right)\left(c_{1}-c_{3}\right)\left(c_{2}-c_{3}\right) \neq 0$, i.e.

$$
d \neq 0, \quad \sigma c_{1}+1-c_{1} \neq 0, \quad \sigma c_{1}+1-c_{2} \neq 0 .
$$

The coefficient $\widehat{a}_{12}$ is polynomic in $\sigma$. Consequently, the matrices $\widehat{A}(\sigma)$ and $A(\sigma)$ are uniformly bounded for any bounded set $J_{\sigma}$. The other coefficients are rational functions of $\sigma$. Remark that the factor $d$ in $\widehat{r}_{21}$ can be eliminated, since $c_{3}-c_{4}=\sigma\left(c_{1}-c_{2}\right)$. This means that $\widehat{B}(\sigma)$ and $\widehat{R}(\sigma)$ cannot be bounded for any choice of $J_{\sigma}$, but they will be for carefully chosen $J_{\sigma}$.

For the above method, $c_{2}-c_{1}=d_{\max }$ and the forbidden stepsize ratios will depend on the selection of the node $c_{1}$. Thus, taking $c_{1}=0, c_{2}=d_{\max }$, which is a natural selection, the method can be extended to variable stepsize for all bounded $\sigma$. More specifically, taking for example $J=[0,3]$, it is easy to see that

$$
\begin{aligned}
& \max _{i, j \leq 2}\left(\left|a_{i, j}(\sigma)\right|\right)=0.920 \text { for all } \sigma, \\
& \max _{\sigma \in J} \max _{i, j \leq 2}\left(\left|b_{i, j}(\sigma)\right|\right)=\max _{i, j \leq 2}\left(\left|b_{i, j}(3)\right|\right)=88.609, \\
& \max _{\sigma \in J} \max _{i, j \leq 2}\left(\left|r_{i, j}(\sigma)\right|\right)=\max _{i, j \leq 2}\left(\left|r_{i, j}(3)\right|\right)=32.384 .
\end{aligned}
$$

For $c_{2}=1, c_{1}=1-d_{\max }$, the method can be extended to variable stepsize for all $\sigma$ except for $\sigma_{1}=\left(c_{1}-1\right) / c_{1}=5.76779$. In this case, for $J=[0,3]$,

$$
\begin{aligned}
& \max _{\sigma \in J} \max _{i, j \leq 2}\left(\left|a_{i, j}(\sigma)\right|\right)=\max _{i, j \leq 2}\left(\left|a_{i, j}(0)\right|\right)=1.0 \\
& \max _{\sigma \in J} \max _{i, j \leq 2}\left(\left|b_{i, j}(\sigma)\right|\right)=\max _{i, j \leq 2}\left(\left|b_{i, j}(3)\right|\right)=25.810 \\
& \max _{\sigma \in J} \max _{i, j \leq 2}\left(\left|r_{i, j}(\sigma)\right|\right)=\max _{i, j \leq 2}\left(\left|r_{i, j}(3)\right|\right)=13.038
\end{aligned}
$$

In both cases, the method can be extended to variable stepsize for practical sequences of the stepsize.

\section{Peer methods with $s=3$}

With 3 stages, we can construct methods of order $2 s-1=5$, expressed in terms of the parameters $p_{32}, d_{2}=c_{2}-c_{1}$ and $d_{3}=c_{3}-c_{1}$. According to Lemma (1) the first and last rows of $\widehat{A}, \widehat{B}$ and $\widehat{R}$ are uniquely defined in terms of the free parameters. On the other hand, for the second stage, the matrices $Q^{(2)}$ and $P^{(2)}$ from (25) are

$$
P^{(2)}=\left(-1-p_{32}, p_{32}, 1\right), \quad Q^{(2)}=\left(u\left(c_{1}\right), u\left(c_{2}\right), u\left(c_{3}\right)\right)^{T}
$$

with $u(x)=\int_{0}^{x}\left(t-c_{1}\right)\left(t-c_{2}\right)\left(t-c_{3}\right) \mathrm{d} t$, and

$\operatorname{det}\left[P^{(2)} Q^{(2)}\right]=d_{3}^{3}\left(3 d_{3}^{2}-5 d_{3}-5 d_{2} d_{3}+10 d_{2}\right)+p_{32} d_{2}^{3}\left(3 d_{2}^{2}-5 d_{2}-5 d_{2} d_{3}+10 d_{3}\right)$.

According to Theorem 2, the second row of $\widehat{A}, \widehat{B}$ and $\widehat{R}$ is uniquely determined in terms of the free parameters whenever

$$
p_{32} \neq-\frac{d_{3}^{3}\left(3 d_{3}^{2}-5 d_{3}-5 d_{2} d_{3}+10 d_{2}\right)}{d_{2}^{3}\left(3 d_{2}^{2}-5 d_{2}-5 d_{2} d_{3}+10 d_{3}\right)} .
$$


The free parameters $p_{32}, d_{2}$ and $d_{3}$ can be chosen to maximize the stability interval and minimize the coefficients of the leading term of the local error. An analysis similar to the case $s=2$ should be carried out, but now, with three degrees of freedom, only a numerical study can be done.

If we restrict ourselves to values for $d_{2}$ and $d_{3}$ such that $\left\{c_{1}, c_{2}, c_{3}\right\} \in[0,1]$, then only methods with small stability regions can be obtained and for such methods $d_{2}$ or $d_{3}$ should be in the neighbourhood of 1 , but in case $d_{2}$ or $d_{3}$ equals 1 the interpolation problem has no solution. For instance, for $p_{32}=$ $1.5, d_{2}=0.55$ and $d_{3}=0.9$ the method has a stability region as is shown in Figure 2 and $\mathbf{C}_{6}=\left(0.28125 \cdot 10^{-5}, 0.867177 \cdot 10^{-3},-0.7111306 \cdot 10^{-3}\right)$, such that $\left\|\mathbf{C}_{6}\right\|_{2}=0.112147 \cdot 10^{-2}$.

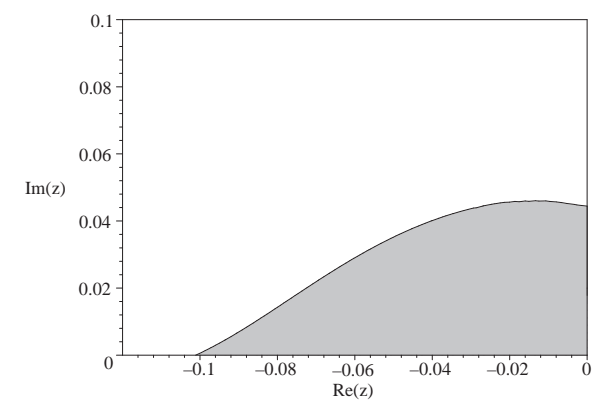

Figure 2: Stability region for the case where $p_{32}=1.5, d_{2}=0.55$ and $d_{3}=0.9$.

After extensive numerical search (see e.g. Figure 3) where we take into account the length of the stability intervals along the real and imaginary axes and also the value of the error constants, we have found that for $p_{32}=-0.522, d_{2}=$ 0.904 and $d_{3}=1.141$ the corresponding fifth order method has a stability interval $[-2.02,0]$ and an interval at the imaginary axis of $[-0.24,0.24]$. Figure 4 , which plots this stability region, shows however that along the imaginary axis, stability is obtained in practice in the interval $[-0.6,0.6]$. For this method, $\mathbf{C}_{6}=\left(.254477 \cdot 10^{-6}, .305637 \cdot 10^{-2}, .198147 \cdot 10^{-2}\right)$ such that $\left\|\mathbf{C}_{6}\right\|_{2}=.364247 \cdot 10^{-2}$. In Table 1 we give the coefficients, 16 figures accurate, of this method.

Larger stability intervals can be obtained taking larger values of $d_{2}$ and $d_{3}$. For example, for $p_{32}=-1.45, d_{2}=1.1$ and $d_{3}=2.9$, the interval along the real axis is $[-2.40,0]$, but the value of $\left\|\mathbf{C}_{6}\right\|_{2}$ is about 40 times larger than for the other method.

Let us analyze now the extension of the method to variable stepsize. Taking $c_{1}=0, c_{2}=0.904, c_{3}=1.141$ and $p_{32}=-0.522$ the method can be extended to variable stepsize except for $\sigma_{1}=\left(c_{3}-1\right) / c_{2}=0.155973$ More specifically, 


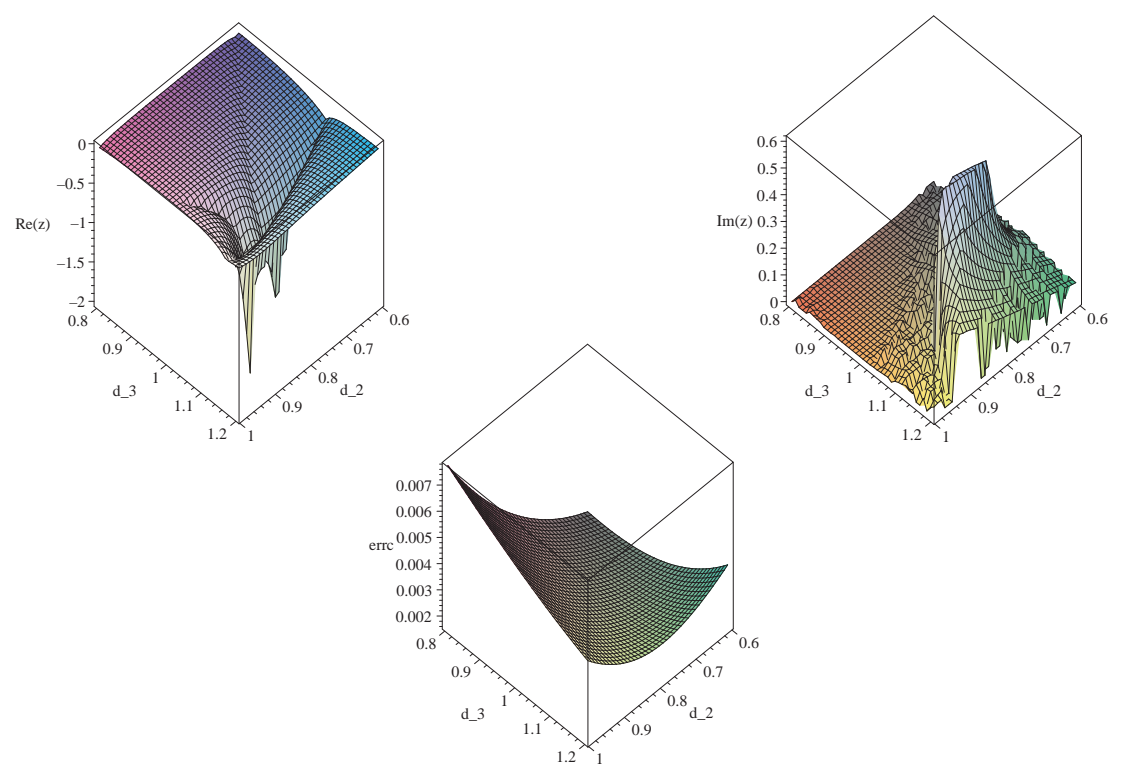

Figure 3: Stability intervals along the real axis (top, left) and along the imaginary axis (top, right) and the value of errc $=\left\|\mathbf{C}_{6}\right\|_{2}$ for $p_{32}=-0.522$.

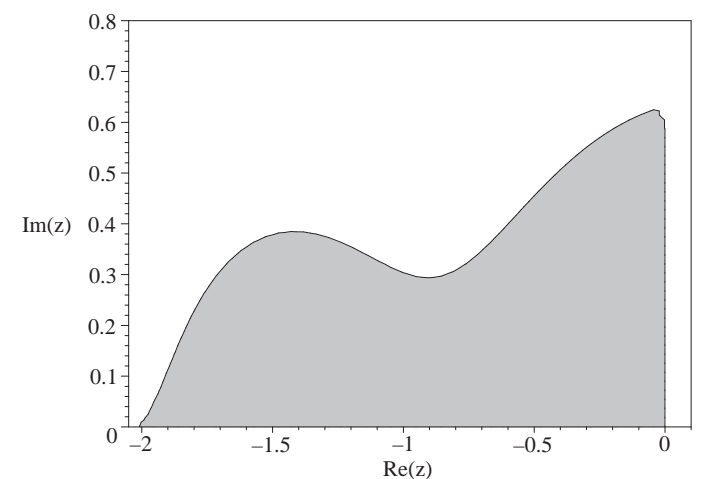

Figure 4: Stability region for the three-stage peer method with $p_{32}=-0.522, d_{2}=0.904$ and $d_{3}=1.141$ 
Table 1: Three-stages peer method with order $5, p_{32}=-0.522, d_{2}=0.904, d_{3}=1.141$.

$$
\begin{aligned}
& a_{11}=.8550915032094356 \cdot 10^{-3}, \quad b_{11}=.17221562082482 \cdot 10^{-3} \text {, } \\
& a_{12}=.6920062545834602, \quad b_{12}=.4157917858290455 \cdot 10^{-1} \text {, } \\
& a_{13}=.3071386539133304, \quad b_{13}=-.1777025246226498 \cdot 10^{-1} \text {, } \\
& a_{21}=5.040475668342306, \quad b_{21}=1.11675014341160, \\
& a_{22}=6.195524959834524, \quad b_{22}=41.79901177123005, \\
& a_{23}=-10.23600062817683, \quad b_{23}=21.92218031561608, \\
& a_{31}=2.631537032613216, \quad b_{31}=.593029841197872, \\
& a_{32}=3.564843018724515, \quad b_{32}=20.47703416241365, \\
& a_{33}=-5.196380051337731, \quad b_{33}=10.66647071584238 \text {, } \\
& r_{21}=-56.85542007719709 \text {, } \\
& r_{31}=-27.35949528575123 \\
& r_{32}=.4704121159473891 \text {. }
\end{aligned}
$$

taking for example $J=[0,2] \backslash(0.15,0.16)$, it can be seen that

$$
\begin{aligned}
& \max _{\sigma \in J} \max _{i, j \leq 3}\left(\left|a_{i, j}(\sigma)\right|\right)=\max _{i, j \leq 3}\left(\left|a_{i, j}(2)\right|\right)=254.010 \\
& \max _{\sigma \in J} \max _{i, j \leq 3}\left(\left|b_{i, j}(\sigma)\right|\right)=\max _{i, j \leq 3}\left(\left|b_{i, j}(2)\right|\right)=831.929 \\
& \max _{\sigma \in J} \max _{i, j \leq 3}\left(\left|r_{i, j}(\sigma)\right|\right)=\max _{i, j \leq 3}\left(\left|r_{i, j}(2)\right|\right)=523.363
\end{aligned}
$$

With this selection of the coefficients, the stepsize control algorithm must avoid the values of $\sigma_{n}$ close to $\sigma_{1}$, which correspond to strong reductions of the stepsize, taking $\sigma_{n}=0.15$ when this happens.

For $c_{1}=-0.141, c_{2}=0.763, c_{3}=1$ and $p_{32}=-0.522$ the method can be extended to variable stepsize for all $\sigma$ except for $\sigma_{1}=\left(c_{2}-1\right) / c_{1}=1.6808, \sigma_{2}=$ 5.57297 and $\sigma_{3}=\left(c_{1}-1\right) / c_{1}=8.0922$ In this case, for $J=[0,2] \backslash(1.6,1.8)$,

$$
\begin{aligned}
& \max _{\sigma \in J} \max _{i, j \leq 3}\left(\left|a_{i, j}(\sigma)\right|\right)=\max _{i, j \leq 3}\left(\left|a_{i, j}(2)\right|\right)=247.480 \\
& \max _{\sigma \in J} \max _{i, j \leq 3}\left(\left|b_{i, j}(\sigma)\right|\right)=\max _{i, j \leq 3}\left(\left|b_{i, j}(1.8)\right|\right)=2146.400 \\
& \max _{\sigma \in J} \max _{i, j \leq 3}\left(\left|r_{i, j}(\sigma)\right|\right)=\max _{i, j \leq 3}\left(\left|r_{i, j}(1.6)\right|\right)=1289.670
\end{aligned}
$$

With this selection of the coefficients, the stepsize control algorithm must avoid the values of $\sigma$ close to $\sigma_{1}=1.16808$, which correspond to reasonable increasing of the stepsize, limiting it to $\sigma=1.16$ unless it is larger than 1.18. In any case, this is not a dramatic limitation.

\section{Numerical experiments}

To show the performance of the peer methods above developed we present here the results obtained with the following two problems: 
- Kepler's problem

$$
p_{i}^{\prime}=-\frac{q_{i}}{\left(q_{1}^{2}+q_{2}^{2}\right)^{3 / 2}}, q_{i}^{\prime}=p_{i}, i=1,2,
$$

with initial conditions

$$
p_{1}(0)=0, p_{2}(0)=\sqrt{\frac{1+e}{1-e}}, q_{1}(0)=1-e, q_{2}(0)=0,
$$

where $0 \leq e<1$ is the eccentricity of the orbit. It is well known that its solution is periodic with period $T=2 \pi$. We have taken $e=0.5$ and $[0,4 T]$ as integration interval.

- The Euler equations that describe the motion of a free rigid body

$$
\frac{d}{d t}\left(\begin{array}{l}
y_{1} \\
y_{2} \\
y_{3}
\end{array}\right)=\left(\begin{array}{ccc}
0 & \omega_{3} y_{3} & -\omega_{2} y_{2} \\
-\omega_{3} y_{3} & 0 & \omega_{1} y_{1} \\
\omega_{2} y_{2} & -\omega_{1} y_{1} & 0
\end{array}\right)\left(\begin{array}{l}
y_{1} \\
y_{2} \\
y_{3}
\end{array}\right)
$$

where $\omega_{j}^{-1}=I_{j}>0$ are the principal momenta of inertia and $y=$ $\left(y_{1}, y_{2}, y_{3}\right)^{T}$ is the angular momentum. We have taken $\omega_{1}=1, \omega_{2}=$ $1-0.51 / \sqrt{1.51}, \omega_{3}=1+1 / \sqrt{1.51}$ together with the initial conditions $y_{1}(0)=0, y_{2}(0)=1$ and $y_{3}(0)=1$. For these conditions, the solution is periodic with period $T=7.45056320933097 \ldots$ The integration interval considered was also $[0,4 T]$.

We have selected in our experiments two peer methods:

- The two-stage third order formula with optimal stability region, given in (37) with $c_{1}=0$.

- The three-stage fifth order formula given in Table 1 , with $c_{1}=0$.

In all the cases we have used a fixed stepsize, taking $h=T / N$ for $N=m 2^{i}$, $i=0,1, \ldots$, with $m$ depending on the order of the method and the problem being integrated, in such a way that for $h=T / m$ the global error is smaller than, but not far from 1. For example, for the Kepler problem integrated with the fifth order formulas we have taken $m=20$. To start the integration, the stages corresponding to the first step were defined as the exact solution, that is, $Y_{0, k}=\left(y\left(t_{0}+c_{1} h\right)^{T}, \ldots, y\left(t_{0}+c_{s} h\right)^{T}\right)^{T}$.

For each integration we have computed at each step the global error, measured in the Euclidean norm, and we have obtained the maximum of these values, GE. We have also computed the number of function evaluations required NFCN at each integration.

First in order to verify numerically the order of the methods we plot the values of the global errors versus the stepsizes used, in double logarithmic scale. In Figure 5 we can see clearly that the numerical order agrees with the theoretical one for both methods. 

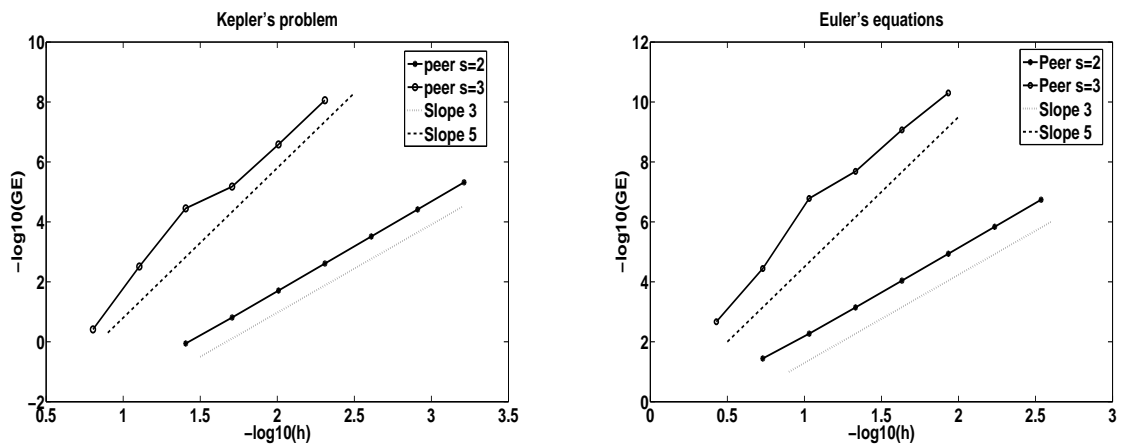

Figure 5: Numerical order of the peer methods of orders 3 and 5.
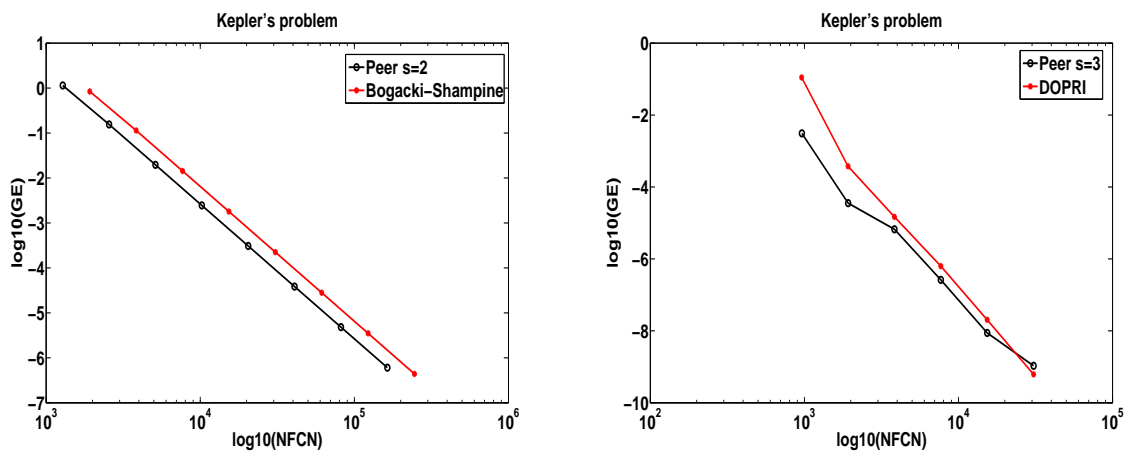

Figure 6: Efficiency plot with the Kepler's problem for the peer method of order 3 and the Bogacki-Shampine RK method of order 3 (left) and for the peer method of order 5 and the DOPRI RK method (right).

Next, to test the efficiency of the methods we have compared them with standard RK methods of the same order. On one side we have considered the Bogacki and Shampine RK method of order 3 [1], whose (2-norm of the) coefficient of the leading term of the local error is 0.0418111 and its stability interval is $[-2.51,0]$. On the other side, we have considered the well known Dormand and Prince RK method [4], that has an error coefficient $0.399080 \cdot 10^{-3}$ and $[-3.31,0]$ as stability interval. Both RK methods have been used in the ODE suit [14] in MATLAB.

In Figure 6 we display the efficiency plots, $\log _{10}(G E)$ versus $\log _{10}$ NFCN, corresponding to the Kepler problem. It can be observed that the peer methods are slightly more efficient than the RK methods in both cases.

In Figure 7 we display the efficiency plots corresponding to the Euler equations. It can be observed that the peer method of order three has a behaviour similar to the one of Bogacki-Shampine formula, whereas the peer method of order five seems to be slightly more efficient than DOPRI. 

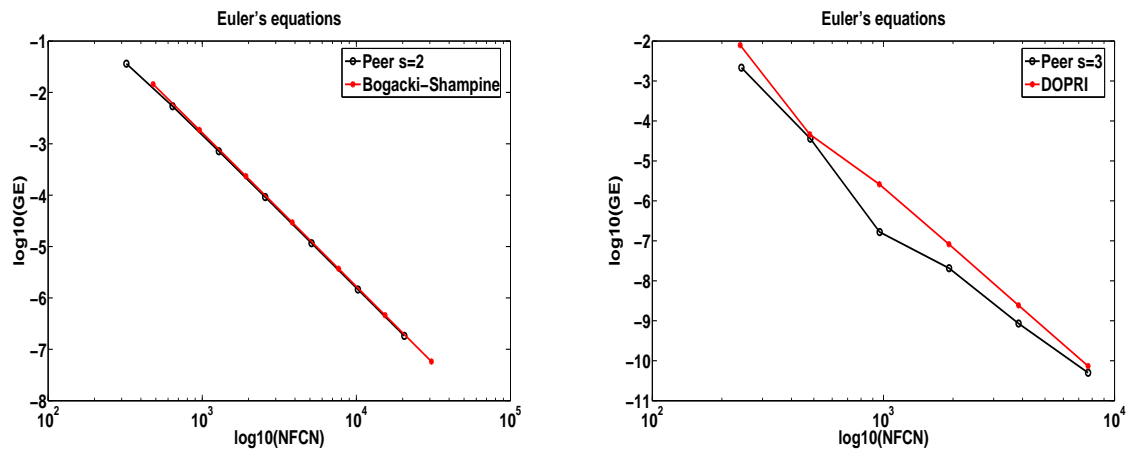

Figure 7: Efficiency plot with the Euler's equations for the peer method of order 3 and the Bogacki-Shampine RK method of order 3 (left) and for the peer method of order 5 and the DOPRI RK method (right).

In order to analyse these results, we must take into account that, in the case of the third order methods, the peer formula requires two stages per step whereas the RK formula requires three. Thus, if we want to compare them at equal computational cost, we must scale the error coefficient of the peer method by the factor $(2 / 3)^{4}$ and its stability interval by $3 / 2$. This gives us 0.028 and $[-3.6,0]$ for the peer versus 0.0418111 and $[-2.51,0]$ for the RK. This can explain why the performance is slightly better for the peer method.

Regarding the fifth order methods, the DOPRI formula needs six function evaluations per step, whereas the peer method requires only 3 . Then we must scale the error coefficient of the peer method by $1 / 2^{6}$ and its stability interval by a factor of 2 . This gives us $0.569136 \cdot 10^{-4}$ and $[-4.04,0]$ for the peer method versus $0.399080 \cdot 10^{-3}$ and $[-3.31,0]$ for the DOPRI, which can explain the better performance of the peer method.

\section{Conclusions}

We have shown that fixed stepsize stable explicit two step peer methods with $s$ stages can attain order $2 s-1$ and we have studied the extension of these methods to variable stepsize. We have also obtained particular methods with $s=2,3$ and orders 3 and 5 respectively. We have finally presented some numerical experiments, showing that peer methods with optimal order can be competitive with standard RK formulas with the same order. Further research on variable stepsize implementations of these methods is in progress.

\section{Acknowledgements}

The authors thank the referees for the careful reading of the manuscript and their helpful suggestions. 


\section{References}

[1] P. Bogacki, L.F. Shampine, A 3(2) pair of Runge-Kutta formulas, Applied Mathematics Letters 2 (4), 321-325 (1989).

[2] J.C. Butcher, The Numerical Analysis of Ordinary Differential Equations: Runge Kutta and General Linear Methods Wiley, New York, (1987).

[3] N.H. Cong, H. Podhaisky, R. Weiner, Numerical experiments with some explicit pseudo two-step RK methods on a shared memory computer, Comput. Math. Appl. 36 (1998) 107-116.

[4] J. Dormand, P. Prince, A family of embedded Runge-Kutta formulae, J. Comput. Appl. Math. 6, 19-26 (1980).

[5] F. R. Gantmacher, The Theory of Matrices, volume two (Chelsea publishing company, New York, 1960).

[6] E. Hairer, S.P. Nørsett and G. Wanner, Solving Ordinary Differential Equations I, Nonstiff Problems (Second Revised Edition, Springer-Verlag, Berlin, 1993).

[7] Z. Jackiewicz, M. Zennaro, Variable-stepsize explicit two-step Runge-Kutta methods, Math. Comput. 59 (1992) 421-438.

[8] Z. Jackiewicz, S. Tracogna, A general class of two-step Runge-Kutta methods for ordinary differential equations, SIAM J. Numer. Anal. 32 (1995) 1390-1427.

[9] S. Jebens, O. Knoth, R. Weiner, Explicit Two-Step Peer Methods for the Compressible Euler Equations. Monthly Weather Review Vol. 137, No. 7, 2380-2392 (2009).

[10] G. Yu. Kulikov and R. Weiner, Doubly quasi-consistent parallel explicit peer methods with built-in global error estimation, J. Comput. Appl. Math., 233, 2351-2364 (2010).

[11] B.A. Schmitt, R. Weiner, Parallel two-step $W$-methods with peer variables, SIAM J. Numer. Anal. 42 (2004), no. 1, 265-282 .

[12] B.A. Schmitt, R. Weiner, K. Erdmann, Implicit parallel peer methods for stiff initial value problems, Appl. Numer. Math. 53 (2005), no. 2-4, 457-470.

[13] B.A. Schmitt, R. Weiner, S. Jebens, Parameter optimization for explicit parallel peer two-step methods. Appl. Numer. Math. 59, 769-782 (2009).

[14] L.F. Shampine, M.W. Reichelt, The Matlab ODE suite. SIAM J. Sci. Comput. 18, 1 (1997) 1-22.

[15] S. Tracogna and B. Welfert, Two-step Runge-Kutta methods: Theory and practice, BIT 40 (2000) 775-799.

[16] H. Podhaisky, R. Weiner, B.A. Schmitt, Linearly-implicit two-step methods and their implementation in Nordsieck form, Appl. Numer. Math. 56 (2006), no. 3-4, 374-387. 
[17] R. Weiner, B.A. Schmitt, H. Podhaisky, S. Jebens, Superconvergent explicit twostep peer methods, J. Comput. Appl. Math., 223, 753-764 (2009).

[18] R. Weiner, K. Biermann, B.A. Schmitt, H. Podhaisky, Explicit two-step peer methods, Comput. Math. Appl. 55, 609-619 (2008). 